TITLE:

\title{
Photothermal processes on a fast time scale: A small molecule and a biological protein (invited)
}

$\operatorname{AUTHOR}(S)$ :

Miyata, R; Terazima, M

\section{CITATION:}

Miyata, R ...[et al]. Photothermal processes on a fast time scale: A small molecule and a biological protein (invited). REVIEW OF SCIENTIFIC INSTRUMENTS 2003, 74(1): 884-888

\section{ISSUE DATE:}

2003-01

URL:

http://hdl.handle.net/2433/49905

\section{RIGHT:}

Copyright 2003 American Institute of Physics. This article may be downloaded for personal use only. Any other use requires prior permission of the author and the American Institute of Physics. 


\title{
Photothermal processes on a fast time scale: A small molecule and a biological protein (invited)
}

\author{
Ryoji Miyata and Masahide Terazima ${ }^{\text {a) }}$ \\ Department of Chemistry, Graduate School of Science, Kyoto University, Kyoto 606-8502, Japan
}

(Presented on 27 June 2002)

\begin{abstract}
The thermalization rate after the relaxation of a photoexcited ionic molecule [malachite green (MG)] and of a biological protein [deoxymyoglobin $(\mathrm{Mb})$ ] were measured by using the acoustic peak delay method of the transient grating technique. It is found that the rate significantly depends on the concentration of MG; the peak delay time decreases with increasing the concentration. This unexpected concentration dependence is explained in terms of the ionic interaction between MG and the counterions. After photoexcitation of $\mathrm{Mb}$, a negative peak delay was observed and interpreted in terms of an interference between the acoustic waves created by a transient thermal expansion of the protein part and by the normal thermal expansion of the medium. (C) 2003 American Institute of Physics. [DOI: 10.1063/1.1516252]
\end{abstract}

\section{INTRODUCTION}

Heating of a sample after photoexcitation of molecules in a condensed phase (photothermal effect) is of major importance not only in chemistry but also in other fields of science, such as laser ablation in industrial technology or biology, and has been a subject of extensive research during the last two decades. The excess energy due to the nonradiative transition between electronic states (internal conversion and/or intersystem crossing) will be first transferred to several energy-accepting vibrational modes, so that the total energy is conserved before and after the transition. The energy ultimately goes to the translational freedom (heating) by a subsequent relaxation process. The relaxation process is mostly induced by molecular collision. This collisional energy relaxation from vibrationally excited polyatomic molecules has been a major topic in physical chemistry for a long time. ${ }^{1,2}$ However, time-resolved studies of the heating process of the matrix due to energy transfer have been very limited because of the lack of experimental techniques. In order to trace the time evolution of the solution temperature in the picosecond time region, we have developed several new methods: temperature lens, ${ }^{3}$ temperature grating, ${ }^{4}$ molecular heater-molecular thermometer system, ${ }^{5}$ and the acoustic peak delay method based on the transient grating technique. $^{6-9}$ In particular, we have demonstrated that the acoustic peak delay method is useful to investigate the energy transfer process and have revealed some prominent features in the thermalization process. For example, in many organic solvents, the process is described by roughly two kinetics: fast $(<1 \mathrm{ps})$ and slow $(20-30 \mathrm{ps})$. This biphase kinetics has been explained by a simple thermalization model; the initial fast process represents the energy transfer to several doorway molecules among the first solvent shell molecules and the slower one represents the thermal diffusion process from the hot solvent molecules to the bulk solvent molecules.

${ }^{a)}$ Electronic mail: mterazima@ kuchem.kyoto-u.ac.jp
The molecules we have investigated so far using these techniques were electrically neutral and relatively small molecules. Here, we report the temperature rise process of the medium after the deactivation of the electronically excited state of a charged molecule [malachite green (MG)] in some organic solvents and of a biological protein [deoxymyoglobin $(\mathrm{Mb})$ ] in aqueous solution (Fig. 1). We may expect that features of the thermalization process could be different from the other systems due to the different intermolecular interaction. The excited states of both molecules relax to the ground state very quickly. (The lifetime of the $S_{1}$ state of MG depends on the viscosity of the solvent and is in a range of $0.3-0.8$ ps in the solvents we examined. ${ }^{10,11}$ It has been reported that the excited states of $\mathrm{Mb}$ relax to the ground state within 3 ps. $^{12}$ ) This fast relaxation ensures that the deactivation rate from the electronically excited state cannot be the rate determining step of the thermalization process.

\section{PRINCIPLE AND METHOD}

When two laser beams are crossed at one spot within the coherence time, an optical interference pattern is created. The spatial modulation of the refractive index or absorption induced by the interference pattern can be detected as the diffraction of another probe light [transient grating (TG) signal]. ${ }^{6-9,13}$

One of the dominant contributions to the TG signal comes from the change of the refractive index caused by the variation of the density, which is induced by the thermal energy deposited into the medium by the intermolecular energy transfer from the excited state. Due to the periodic excitation in space, the medium expands periodically and the signal due to this refractive index change oscillates sinusoidally (frequency $\omega=v q ; v$, speed of sound in the solution; $q$ : grating wave number) (acoustic TG signal). Because of the convolution effect, the acoustic oscillation is gradually distorted as the heat dissipation rate becomes slow. Even though the thermalization rate can be obtained from a fitting of the whole time profile of the acoustic signal, the time 


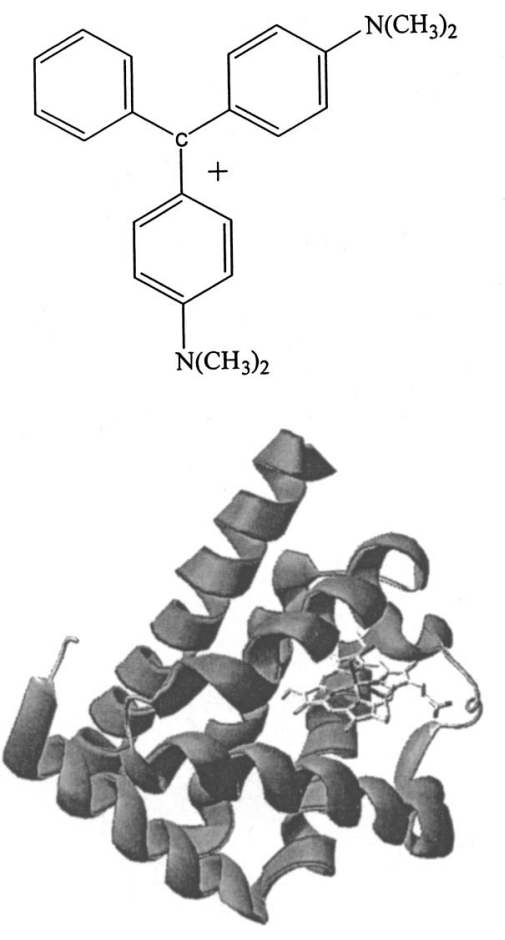

$1 / 2\left|\left(\mathrm{COO}^{-}\right)_{2} 2 \mathrm{C}_{2} \mathrm{H}_{2} \mathrm{O}_{4}\right|$

FIG. 1. Molecular structures of malachite green and myoglobin.

resolution of the curve fitting method is not high enough for studying the fast thermalization processes. On the other hand, by monitoring the peak delay time $\left(\tau_{\text {pd }}\right)$ from the impulsive thermalization case, the time delay of the thermalization can be detected with a high time resolution. We measured the acoustic frequency $(\omega)$ and the temporal delay of the acoustic wave from the time difference between the first $\left(t_{1}\right)$ and the second $\left(t_{2}\right)$ acoustic peaks. $\tau_{\text {pd }}$ was calculated by a relation of $\tau_{\mathrm{pd}}=\left(3 t_{1}-t_{2}\right) / 2$. When acoustic damping is neglected, the acoustic peak delay time $\left(\tau_{\mathrm{pd}}\right)$ is almost the same as the thermalization time. ${ }^{6-9}$ This is the principle of the acoustic delay method.

With increasing the attenuation constant, the peak delay decreases. ${ }^{6}$ When there are two heat releasing processes with different lifetimes of $\tau_{f}$ and $\tau_{s}\left(\tau_{f}<\tau_{s}\right)$, the temporal profile of the acoustic signal should depend on these time constants as well as the releasing energy from these processes. If we calculate the peak delay as a function of $Q_{s} / Q_{\text {tot }}\left[Q_{s}\right.$; thermal energy from the slower process, $Q_{\text {tot }}\left(=Q_{s}+Q_{f}\right)$; total thermal energy], the acoustic peak shifts linearly with $Q_{s} / Q_{\text {tot }}$ from $\tau_{\mathrm{pd}}$ for $Q_{\mathrm{tot}}=Q_{f}$ to $\tau_{\mathrm{pd}}$ for $Q_{\mathrm{tot}}=Q_{s}$. We have shown that $\tau_{\mathrm{pd}}$ provides the energy weighted average thermalization time. ${ }^{6}$ For example, if there are two thermalization processes with lifetimes of $\tau_{f}$ and $\tau_{s}, \tau_{\mathrm{pd}}$ is given by

$$
\tau_{\mathrm{pd}}=\left[Q_{f} /\left(Q_{f}+Q_{s}\right)\right] \tau_{f}+\left[Q_{s} /\left(Q_{f}+Q_{s}\right)\right] \tau_{s},
$$

where $Q_{f}$ and $Q_{s}$ are thermal energies associated with the $\tau_{f}$ and $\tau_{s}$ processes, respectively.

The TG and transient absorption (TA) experimental setup is similar to that reported previously. ${ }^{6-9}$ A pulse from a fiber laser regeneratively amplified by a YAG laser (Clark CPA2001, wavelength $=775 \mathrm{~nm}$ ) was frequency doubled by a BBO crystal and split into two. These pulses were used as excitation beams. These beams were slightly focused by
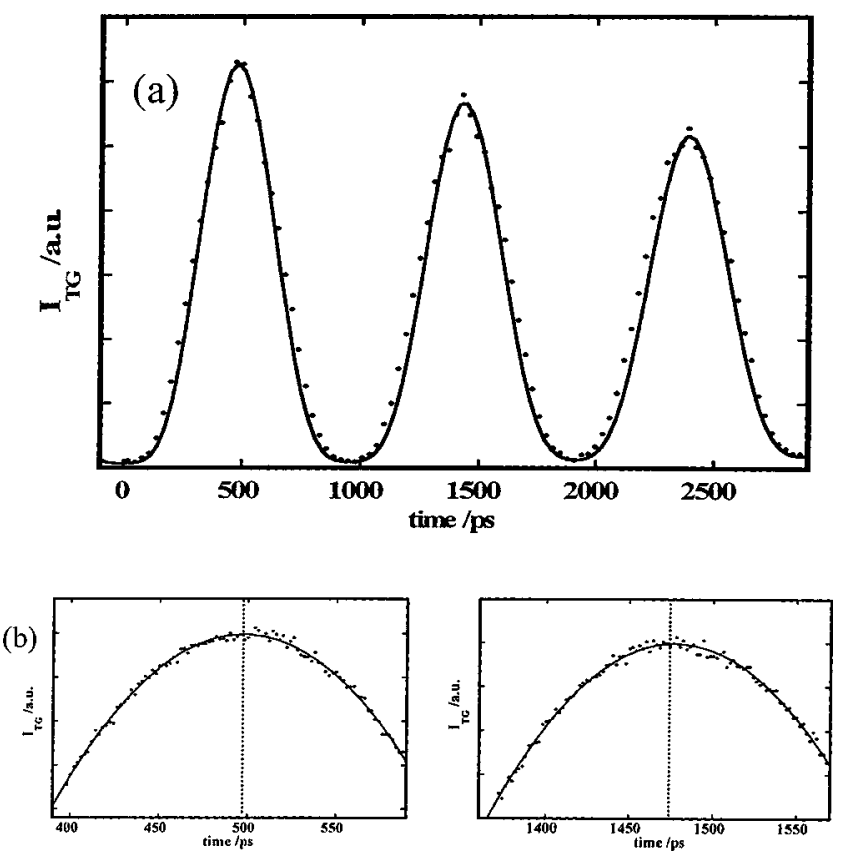

FIG. 2. (a) Acoustic signal after the photoexcitaiton of MG in methanol and (b) the amplified peak parts.

lenses $(f=25 \mathrm{~cm})$ and crossed at about $30^{\circ}$ inside the quartz sample cell in order to generate an optical interference pattern. The fundamental light after the second-harmonic generation crystal was used as a probe beam. The probe beam passed through an optical delay line and was slightly focused by a lens $(f=20 \mathrm{~cm})$ and brought into the sample cell by an angle that satisfied the Bragg condition. The diffracted TG signal was separated from the other beams with a pinhole and a glass filter (Toshiba R-60), and detected by a photomultiplier.

MG from the Exiton Company and Mb from the Nakalai Tesque Company were used without further purification. Solvents were purchased from the Nakalai Tesque Company.

\section{RESULTS AND DISCUSSION}

\section{A. Photothermal process of photoexcited MG}

After the photoexcitation of MG, a weak TG signal appears in a fast time scale ( $0-10 \mathrm{ps}$ range), which must be the population grating $(\mathrm{PG})$ signal representing the dynamics of the excited state. The acoustic signal gradually appears after the PG signal [Fig. 2(a)]. The acoustic signal can be fitted by the thermodynamical equation presented before. ${ }^{6}$ Figure 2(b) shows the top part of the acoustic signal of MG in methanol at $0.75 \mathrm{mM}$. The peak position was determined by the fitting of only the top of the oscillation with a function of $a(t$ $\left.-\tau_{i}\right)^{2}+b$, where $a$ and $b$ are constants related to the sensitivity and $\tau_{i}$ is the time of the first $(i=1)$ and the second $(i=2)$ acoustic peaks [Fig. 2(b)]. From $\tau_{1}$ and $\tau_{2}$, the peak delay time $\tau_{\text {pd }}$ is calculated and $\tau_{\text {temp }}$ is obtained after correcting the effect of acoustic damping. As mentioned in Sec. II, $\tau_{\text {temp }}$ is an energy weighted average of the energy transfer rate from the excited molecules to the solvent molecules. For $0.75 \mathrm{mM} \mathrm{MG} /$ methanol solution, $\tau_{\text {temp }}=8 \pm 2 \mathrm{ps}$ was obtained. The electronically excited state of MG relaxes to the 


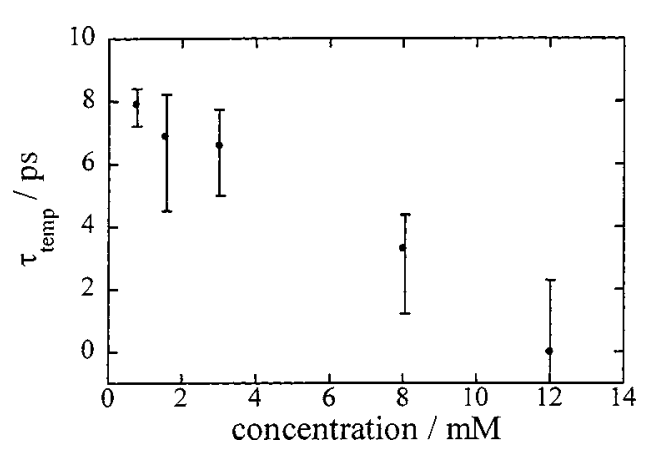

FIG. 3. Concentration dependence of the thermalization time of MG in methanol.

ground state with a lifetime of $0.5 \mathrm{ps}$ in methanol. ${ }^{10}$ Since this time is much shorter than the observed peak delay, the contribution of the excited state relaxation can be neglected and the observed peak delay time should reflect the energy conversion rate from the highly excited vibrational energy in the ground state to the translational energy.

The acoustic peak delay times were measured at various concentrations. Interestingly, we found that $\tau_{\text {temp }}$ depends on the concentration of MG. The rate increases with the concentration of MG in all solvents we examined; methanol, ethanol, and acetonitrile. Figure 3 depicts the concentration dependence of $\tau_{\text {temp }}$ in methanol. For example, $\tau_{\text {temp }}$ is $8 \mathrm{ps}$, at $0.75 \mathrm{mM}$ in methanol, but it becomes $3 \mathrm{ps}$ at $8.1 \mathrm{mM}$.

So far, we have investigated $\tau_{\text {temp }}$ for many compounds such as Betaine-30, ${ }^{8}$ 2-hydroxybenzophenone, ${ }^{7}$ 2-(2' -hydroxy-5' -methylphenyl)benzotriazole, ${ }^{7}$ azobenzene, ${ }^{6}$ and so on. The $\tau_{\text {temp }}$ values of these compounds do not depend on the concentration. The observed concentration dependence in this study is not caused by an experimental artifact from a possible misalignment. We have confirmed that the peak delay time of $p$-nitroaniline in methanol remains $4 \pm 2 \mathrm{ps}$ in the range of $0.1-80 \mathrm{mM}$. Therefore, the concentration dependence of $\tau_{\text {temp }}$ is a unique characteristic of MG.

An important and interesting question is: why does the thermalization time of MG depend on the concentration? An average distance between $\mathrm{MG}$ at $10 \mathrm{mM}$ is about $5.5 \mathrm{~nm}$, which is too long to enhance the thermalization rate by a possible intermolecular interaction between MG. A simple explanation is the cluster formation in the ground state at high concentrations, and the energy transfer rate to the translational mode becomes efficient due to the clusters. Indeed, a cluster formation was observed in aqueous solution from the concentration dependence of the ground state absorption spectrum. We examine the absorption spectrum at various concentrations in the range of $0.01-12 \mathrm{mM}$ in methanol. However, we could not find any evidence for the presence of clusters in the solvents we examined. Hence, aggregate formation is less plausible.

Considering that electrically neutral molecules do not show this concentration dependence, we speculate that the counterion (oxalic ion) around $\mathrm{MG}$ at high concentrations affects the energy relaxation of MG. In order to investigate this possibility, we measured $\tau_{\text {temp }}$ for samples by adding oxalic acid in the solution. Interestingly, $\tau_{\text {temp }}$ decreases to
$2.4 \mathrm{ps}$ when oxalic acid was added to the solution $(28 \mathrm{mM})$. On the other hand, when a neutral molecule, naphthalene, was added into the solution, $\tau_{\text {pd }}$ does not change within experimental uncertainty. This salt effect may support the above suggestion: the thermalization rate is enhanced by the electric interaction between the photoexcited MG and the counterion.

\section{B. Photothermal process of photoexcited Mb}

Similar to $\mathrm{MG}$, after the complete decay of the excited state $(t>3 \mathrm{ps})$ of $\mathrm{Mb}$, a temporally oscillating acoustic grating signal appears. Measuring the peak position carefully, we surprisingly found the acoustic peak delay time after the photoexcitation of deoxyMb to be a negative, $-15 \pm 2 \mathrm{ps}$. We will consider the energy relaxation route from the heme to the solvent from this anomalous peak shift.

The cooling process of the heme has been previously studied by the hot band detection of the band III region. ${ }^{14}$ This research revealed that the temperature of the heme is cooled by time constants of 3.4 and 6.2 ps. Time-resolved Raman scattering experiment of carboxymyoglobin showed that the cooling of the heme is expressed by a biexponential function, 3.0 ps decay with an amplitude of $93 \%$ and 25 ps with $7 \%$ amplitude. ${ }^{15}$ Miller and co-workers detected the pressure wave induced by the ligand photodissociation of carboxymyoglobin and obtained the energy and structural dynamics after the reaction. ${ }^{16}$ However, there is no experimental information on the energy transfer route.

If one considers a fact that energy is deposited to the matrix only after photoexcitation, it is easy to understand that the negative peak delay cannot be expected as the thermalization time. We first qualitatively explain this negative value. Here, we suggest that the interference between two different sources of the acoustic waves is the cause of the negative shift of the wave phase as follows. Following the excited state relaxation, the excess energy is damped to the surrounding protein and/or the water molecules. If the energy that is transferred to the protein part heats up the structure, the protein should experience thermal expansion of the structure. The thermal expansion should launch the pressure wave, which creates the acoustic TG signal due to the protein expansion. At the same time, the energy that is transferred to the solvent heats up the matrix and the thermal expansion of the medium takes place. This expansion also creates the pressure wave. These two waves should interfere with each other and it could create the observed anomalous negative $\tau_{\text {pd }}$.

Next, this interpretation is quantitatively examined. The pressure wave created under a similar condition has been treated theoretically by Chen and Diebold. ${ }^{17}$ They derived an analytical equation for describing the temporal profile of the acoustic wave under a condition that pressure waves are created by the thermal expansion of the medium and the molecular volume change by a chemical reaction. When the excited species evolves instantaneously and decays exponentially with a time constant of $\tau$, the medium density change is given by ${ }^{17}$ 


$$
\begin{aligned}
\delta(\hat{x}, \hat{t})= & u(\hat{t}) \cos \hat{x} \frac{E \alpha}{C_{p}}\left\{\frac{1}{1-\hat{l}_{h} \hat{\tau}}\left[\frac{-\exp \left(\hat{l}_{h} \hat{t}\right)+\exp (-\hat{\gamma} \hat{t})\left[\cos \hat{t}+\left(\hat{\gamma}-\hat{l}_{h}\right) \sin \hat{t}\right]}{1+\left(\hat{\gamma}-\hat{l}_{h}\right)^{2}}\right]+\left[-\frac{1}{1-\hat{l}_{h} \hat{\tau}}+\frac{C_{p} \beta_{c}}{\tilde{h} \alpha}\right]\right. \\
& \left.\times\left[\frac{-\exp (-\hat{t} / \hat{\tau})+\exp (-\hat{\gamma} \hat{t})[\cos \hat{t}+(\hat{\gamma}-1 / \hat{\tau}) \sin \hat{t}]}{1+(\hat{\gamma}-1 / \hat{\tau})^{2}}\right]\right\},
\end{aligned}
$$

where

$$
\begin{aligned}
& \hat{t}=c k t, \quad \hat{\tau}=c k \tau, \quad \hat{x}=k x, \quad \hat{l}_{h}=l_{h} k=D_{t h} k / c, \\
& \hat{\gamma}=\frac{1}{2}\left[\hat{l}_{\nu}^{\prime}+(\gamma-1) \hat{l}_{h}\right], \quad \gamma=C_{p} / C_{\nu}, \quad \hat{l}_{\nu}^{\prime}=\left(\eta+\frac{3}{4} \mu\right) / \rho c .
\end{aligned}
$$

$\alpha$ is the thermal expansion coefficient of the solvent, $C_{p}$ is the heat capacity of the solvent at a constant pressure, $u(t)$ is the Heaviside function, $E$ is the laser energy absorbed per unit volume, $c$ is the sound velocity, $k$ is the grating vector, $D_{\text {th }}$ is the thermal diffusion constant, $\eta$ is the bulk viscosity, $\mu$ is the shear viscosity, and $\rho$ is the density.

For the $\mathrm{Mb}$ case, the previous studies showed that the surrounding water is heated up by a biexponential function. ${ }^{18}$ Here, we assume that the thermal energy of the water matrix is expressed by

$$
Q_{\text {solv }}(t)=Q_{\text {tot }}\left\{1-b_{1} \exp \left(-t / \tau_{b 1}\right)+b_{2} \exp \left(-t / \tau_{b 2}\right)\right\},
$$

where $Q_{\text {tot }}$ is the total energy for heating, which should be equal to the photon energy of the excitation, and $b_{i}(i$ $=1,2$ ) denote the relative amplitudes for the processes with lifetimes of $\tau_{b i}$. We further modeled that the energy transfer from the heme takes place in two routes independently: from the heme to protein (amount of the thermal energy: $Q_{p}$ ) and from the heme to the solvent $\left(Q_{\text {tot }}-Q_{p}\right)$. Since the thermalization process is frequently expressed by a biexponential function, ${ }^{15,18}$ the thermal energy of the heme ( $\left.Q_{\text {heme }}\right)$ is written by

$$
\begin{aligned}
Q_{\text {heme }}(t)= & Q_{p}\left\{a_{1} \exp \left(-t / \tau_{a 1}\right)+a_{2} \exp \left(-t / \tau_{a 2}\right)\right\} \\
& +\left(Q_{\text {tot }}-Q_{p}\right)\left\{b_{1} \exp \left(-t / \tau_{b 1}\right)\right. \\
& \left.+b_{2} \exp \left(-t / \tau_{b 2}\right)\right\} .
\end{aligned}
$$

If a part of the energy of the heme does not go through the protein, but directly into the buffer, $Q_{p} / Q_{\text {tot }}$ should be less than 1.

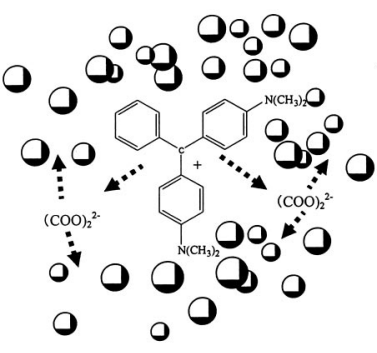

(a)

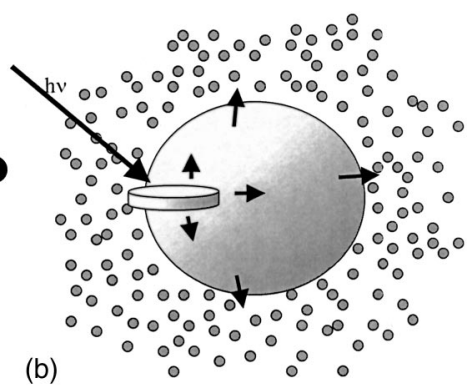

FIG. 4. Thermalization models for (a) MG in methanol and (b) $\mathrm{Mb}$ in aqueous solution. Arrows show the efficient route for the energy conversion process from the photoexcited molecules to the solvent.
First, we consider one of the extreme cases that all of the electronic energy is transferred to the thermal mode of the medium directly, not through the protein. In this case, the acoustic wave should be the same as the case that the electronic energy of a relatively small organic molecule exposed in medium is transferred to the solvent. When we use $b_{1}$ $=0.6, \tau_{b 1}=7.5 \mathrm{ps}, b_{2}=0.4$, and $\tau_{b 2}=20 \mathrm{ps},{ }^{18} \tau_{\mathrm{pd}}$ is calculated to be $12 \mathrm{ps}$. In fact, we have experimentally shown that the peak delay time is in the range of 5-20 ps for most of the organic molecules. ${ }^{6-9}$ Hence, this energy transfer process cannot explain the observed result as long as only heating of the solvent is considered.

Second, we calculate $\tau_{\text {pd }}$ for the other extreme case; all of the excited energy is transferred from the heme to the protein $\left(Q_{p} / Q_{\text {tot }}=1\right)$. The acoustic signal initially shows the expansion wave and then the compressed wave. When the acoustic wave from the medium interferes with this wave, the arrival time of the peak shifts to an early time. Using data of the heme cooling of carboxymyoglobin $\left(a_{1}=0.6, \tau_{a 1}\right.$ $\left.=7.5 \mathrm{ps}, a_{2}=0.4, \tau_{a 2}=20 \mathrm{ps}\right){ }^{7}$ the thermal expansion coefficient $\left(345 \times 10^{-6} \mathrm{~K}^{-1}\right)$ (Ref. 19) and heat capacity (1.36 $\mathrm{J} \mathrm{K}^{-1} \mathrm{~g}^{-1}$ ) (Ref. 20) of Mb, we found that $\tau_{\mathrm{pd}}$ is calculated to be largely negative $(-19.4 \mathrm{ps})$, which is rather close to the experimentally observed value $(-15 \mathrm{ps})$.

If a part of the energy is transferred to the aqueous solution and the protein matrix simultaneously, the expected peak delay time should be the average of the positive and negative values. In order to reproduce the observed $\omega_{\mathrm{pd}}$ by changing $Q_{p} / Q_{\text {tot }}$, we found that about $17 \%$ of the energy should be transferred to the water first. This result strongly supports a mechanism that most of the absorbed photon energy by the heme is first transferred to the protein matrix and then the energy is dissipated to the aqueous solution.

In summary, we studied the thermalization rate after photoexcitation of MG in various solvents and deoxymyoglobin in aqueous solution by the acoustic peak delay method and hot absorption detection. Anomalously large solute concentration dependence was observed for the thermalization rate of MG. The dependence indicates that the energy transfer becomes more efficient with increasing the concentration. The observed concentration dependence was attributed to the electric interaction between the positively charged MG and the counterions (Fig. 4). We also observed a transient thermal expansion of $\mathrm{Mb}$ due to the energy transfer from the photoexcited heme to the protein interior (Fig. 4).

\section{ACKNOWLEDGMENT}

The authors are indebted to Professor Y. Kimura at Kyoto University for his assistance with the experiments. 
${ }^{1}$ D. W. Oxtoby, Adv. Chem. Phys. 47, 487 (1981).

${ }^{2}$ V. E. Bondybey, Annu. Rev. Phys. Chem. 35, 591 (1984).

${ }^{3}$ M. Terazima, J. Chem. Phys. 105, 6587 (1996); Isr. J. Chem. 38, 143 (1998).

${ }^{4}$ T. Okazaki, N. Hirota, and M. Terazima, J. Phys. Chem. A 101, 650 (1997).

${ }^{5}$ T. Okazaki, N. Hirota, T. Nagata, A. Osuka, and M. Terazima, J. Am. Chem. Soc. 121, 5079 (1999); J. Phys. Chem. A 103, 9591 (1999).

${ }^{6}$ M. Terazima, M. Takezaki, S. Yamaguchi, and N. Hirota, J. Chem. Phys. 109, 603 (1998).

${ }^{7}$ T. Okazaki, N. Hirota, and M. Terazima, J. Chem. Phys. 110, 11399 (1999); N. Saga, Y. Kimura, N. Hirota, and M. Terazima, Chem. Phys. Lett. 332, 496 (2000).

${ }^{8}$ M. Terazima, Chem. Phys. Lett. 305, 189 (1999).

${ }^{9}$ M. Terazima, Bull. Chem. Soc. Jpn. 74, 595 (2001).

${ }^{10}$ V. Sundstörm, T. Gillbro, and H. Bergström, Chem. Phys. 73, 439 (1982); V. Sundstörm and T. Gillbro, J. Chem. Phys. 81, 3463 (1984); D. BenAmotz and C. B. Harris, Chem. Phys. Lett. 119, 305 (1985); T. Robl and A. Seilmeier, ibid. 147, 544 (1988); M. Yoshizawa, K. Suzuki, A. Kubo, and S. Saikan, ibid. 290, 43 (1998).

${ }^{11}$ Y. Nagasawa, Y. Ando, and T. Okada, Chem. Phys. Lett. 312, 161 (1999);
Y. Nagasawa, Y. Ando, D. Kataoka, H. Matsuda, H. Miyasaka, and T. Okada, J. Phys. Chem. A 106, 2024 (2002).

${ }^{12}$ J. W. Petrich, C. Poyart, and J. L. Martin, Biochemistry 27, 4049 (1988).

${ }^{13}$ H. J. Eichler, P. Gunter, and D. W. Pohl, Laser-Induced Dynamic Gratings (Springer, Berlin, 1986); M. Terazima, Adv. Photochem. 24, 255 (1998).

${ }^{14}$ M. Lim, T. A. Jackson, and P. A. Anfinrud, J. Phys. Chem. 100, 12043 (1996); T. A. Jackson, M. Lim, and P. A. Anfinrud, Chem. Phys. 180, 131 (1994).

${ }^{15}$ Y. Mizutani and T. Kitagawa, Science 278, 443 (1997).

${ }^{16}$ J. Deàk, H-L. Chiu, C. M. Lewis, and R. J. D. Miller, J. Phys. Chem. B 102, 6621 (1998); L. Richard, L. Genberg, J. Deak, H.-L. Chiu, and R. J. D. Miller, Biochemistry 31, 10703 (1992).

${ }^{17}$ H. Chen and G. J. Diebold, J. Chem. Phys. 104, 6730 (1996).

${ }^{18}$ Y. Lian, B. Locke, Y. Kholodenko, and R. M. Hochstrasser, J. Phys. Chem. 98, 11648 (1994).

${ }^{19}$ H. Frauenfelder, H. Hartmann, M. Karplus, I. D. Kuntz, Jr., J. Kuriyan, F. Parak, G. A. Petsko, D. Ringe, R. F. Tilton, Jr., M. L. Connolly, and N. Max, Biochemistry 26, 254 (1987).

${ }^{20}$ P. L. Privalov, E. I. Tiktopulo, S. Yu. Venyaminov, Yu. V. Griko, G. I. Makhatadze, and N. N. Khechinashvili, J. Mol. Biol. 205, 737 (1989). 